\title{
Development of Management System for eduroam Database Updated Specification
}

\author{
Arthur S. Petrosyan and Gurgen S. Petrosyan \\ Institute for Informatics and Automation Problems of NASRA \\ e-mail: arthur@sci.am, gurgen@sci.am
}

\begin{abstract}
This paper presents a system developed to simplify the eduroam database management. The goal of the eduroam database is to provide the necessary information needed for the operation of the eduroam service and related supporting services like eduroam monitoring, service location maps and usage statistics, as well as the eduroam CAT tool. eduroam database specifications have been updated to the second version and the update has recently become mandatory, requiring many changes for databases of all National Roaming Operators (NRO). The solution presented here can be useful for NRO administrators to simplify the eduroam database management.
\end{abstract}

Keywords: eduroam, NRO, Wi-Fi, Wireless, Monitoring, Statistics, Database.

\section{Introduction}

eduroam (education roaming) [1] has become a well-known secure wireless network roaming access service used by the international research and education community. It enables scientists, researchers, teachers, students from different institutions to securely gain Wi-Fi Internet access at any eduroam-enabled institution worldwide.

The eduroam service organization model assumes that in each country there is a National Roaming Operator (NRO), mostly operated by the National Research and Education Network (NREN) of that country. The eduroam database has been built as a central database with a mechanism that automatically collects data from NROs to enable the operation of the eduroam service and related supporting services like eduroam monitoring, service location maps and usage statistics, as well as the eduroam CAT tool.

eduroam database specifications have been updated to the second version and the update have recently become mandatory [2], requiring many changes for all National Roaming Operators (NRO). This paper presents a system developed for managing the eduroam database with specifications updated to the second version. 


\section{Developed Management System}

According to the new second version (v.2.0) of eduroam database specification [3], each NRO should provide general data in the defined XML or JSON format. The data should be available at the specific, predefined URLs: http://www.eduroam. $<$ tld $>/$ general/<dataset-name $>$ and must be accessible from the eduroam database server site - monitor.eduroam.org. Data for the eduroam database v.2.0 can be validated by XML [4] or JSON validators [5].

The developed Management System is fully compliant with the eduroam database v.2.0 specification and provides a flexible web interface to manage data for a particular NRO. We have chosen to implement only the JSON version in our system and omit the XML format, since one of them is required, not both, and JSON seems to be preferred by the eduroam database server-site monitoring. MySQL/Maria DB was chosen as a backend for the implementation of eduroam database specification. Appropriate MySQL tables were designed and created for datasets (i.e., database tables) and respective fields (attributes) containing general data as described in [3]. The Web interface created for database management provides the possibility to add/edit both the Institutions and Service Locations. The main page of the web interface looks like the one presented in Fig. 1.

\section{eduroamDB}

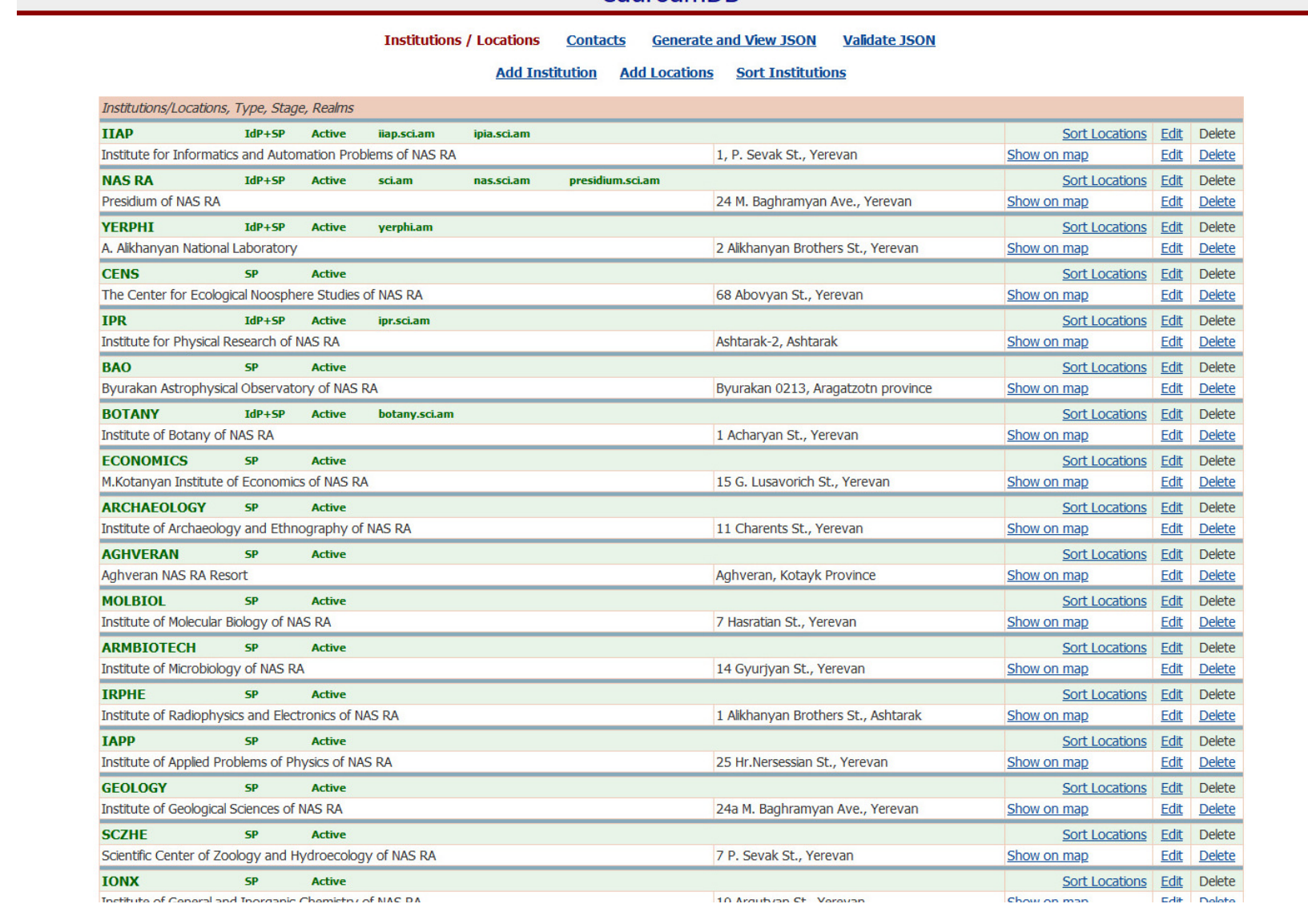

Fig. 1. Main page.

The created web interface is English language-based. Text fields in the database are bilingual (English and Armenian), but only English fields are required. As a result, the system can be used by any NRO worldwide. 
A separate Contact table is created for each Institution, and contact persons can be added from the web interface and then selected as responsible for multiple Institutions (Fig. 2). It is allowed to add up to five contact person per Institution.

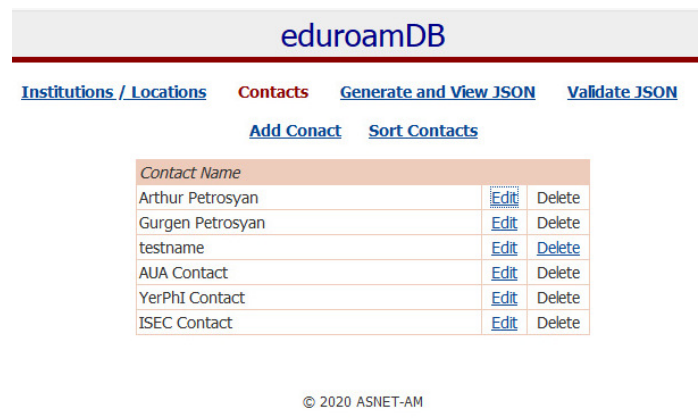

Fig. 2. Contacts page.

Fig. 3.

Next, the Institution can be added by filling in the required fields in the form presented in

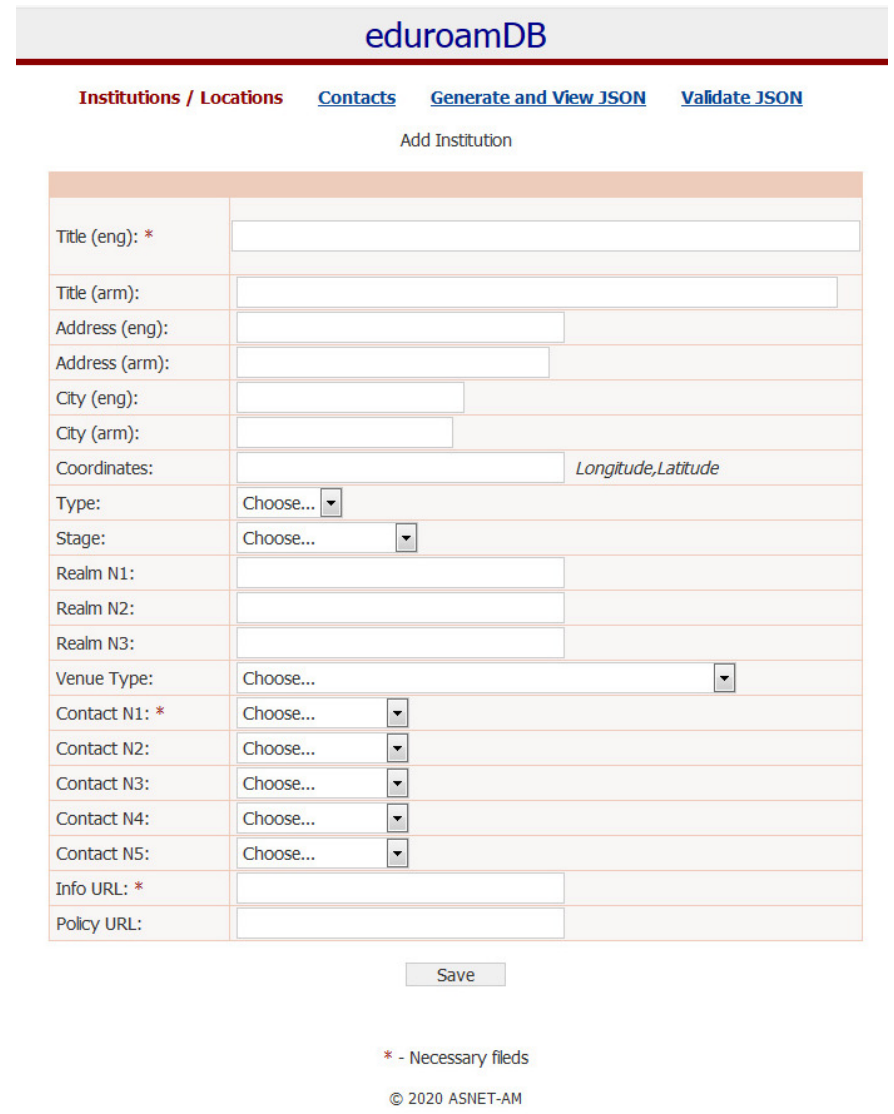

Fig. 3. Institution page.

The locations interface then allows specifying the location data for each registered Institution (Fig. 4). Required fields include location GPS Coordinates. The web interface of our system allows us to easily select a location on the map and put the appropriate coordinates into the form. This makes filling in the data much easier. 


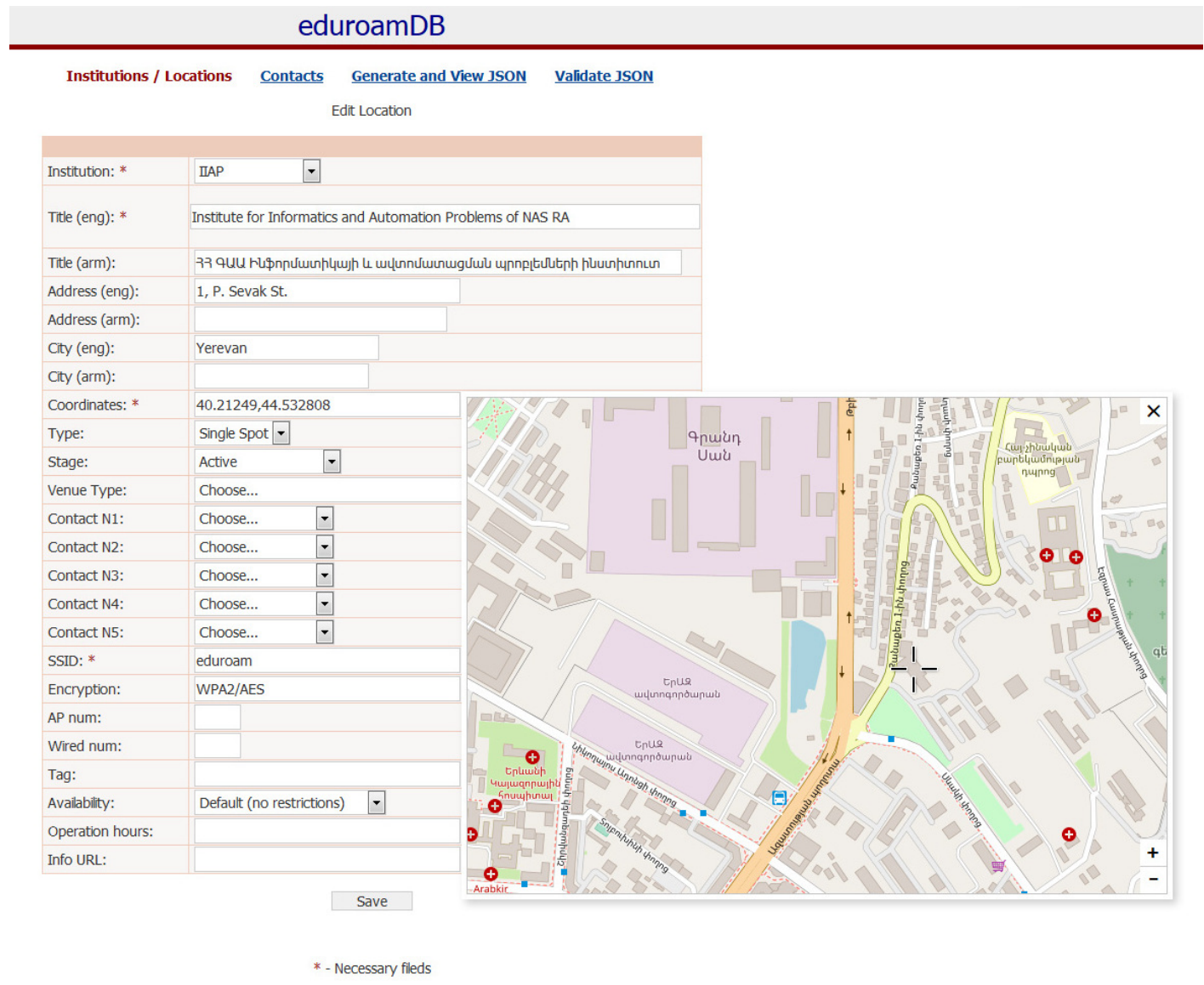

Fig. 4. Locations page.

After having the data of Institutions and Service Locations ready in the database, we can generate a JSON file, as well as validate it with the validators of central eduroam database server site. After generation, the system places the files in a special folder on the web server, the access to which is open only for the IP address of the central monitoring server eduroam, which downloads them from NROs on a daily basis, checks them for compliance with the requirements, and updates them. To prevent data loss, all old previously generated JSON files are stored in a separate backup folder with the date and time of their generation.

All the data can be edited and verified at any time via the web interface. After adding an organization, it is possible to add/edit/delete/sort the eduroam service locations for the organization in accordance with the database structure. In particular, the following fields are available: name, address, city, coordinates, connection status, contact persons, type of service location. In addition, there are specific fields such as: type of Wi-Fi coverage (Single/Area/Mobile), name of the Wi-Fi network (SSID) that in most cases is "eduroam", encryption (WPA, WPA2), number of access points and availability (physical access restrictions or no restrictions).

\section{Service Location Map}

Along with the backend Management System described above, a frontend service location map was also created. It takes data from the same Institutions and Service Locations database and presents it on the NROs website. The current version of it is part of Armenian NRO website [6] and is based on OpenStreetMap [7] and Leaflet [8]. 


\section{Advantages}

Similar eduroam database management systems were developed by Greek NREN GRNET and called DJNRO [9], as well as by Czech NREN CESNET [10]. But the main issue of using this DJNRO is that it has Service locations coordinates support based on the Google Maps API, which has become a paid service since 2018. Instead, in our work we used the free OpenStreetMap/Leaflet [7] [8] alternatives. The CESNET solution turned out to be hard to implement on our site because of many separated scripts and insufficiently documented solution.

\section{Conclusion}

This solution may be interesting for any NROs worldwide. It is currently implemented in ASNET-AM for managing the Armenian part of eduroam database. The solution we presented is all-in-one for NROs functions regarding the management of eduroam database, as well as for presenting this data on the service location map.

\section{References}

[1] Online]. Available: https://www.eduroam.org/

[2] [Online]. Available: https://monitor.eduroam.org/fact_eduroam_db.php

[3] [Online]. Available: https://monitor.eduroam.org/eduroam-database/v2/docs/eduroamdatabase-ver17102017.pdf

[4] [Online]. Available: https://monitor.eduroam.org/eduroamdatabase/v2/scripts/xml_validation_test.php

[5] [Online]. Available: https://monitor.eduroam.org/eduroamdatabase/v2/scripts/json_validation_test.php

[6] [Online]. Available: https://www.eduroam.am/

[7] [Online]. Available: https://www.openstreetmap.org

[8] [Online]. Available: https://leafletjs.com/

[9] [Online]. Available: https://github.com/grnet/djnro

[10] [Online]. Available: https://github.com/CESNET/eduroam-db

Submitted 10.12.2019, accepted 04.05.2020. 


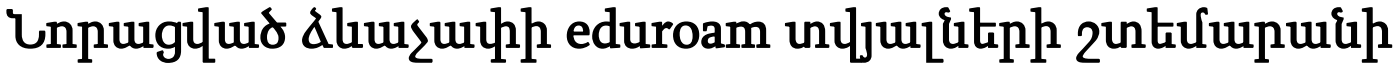

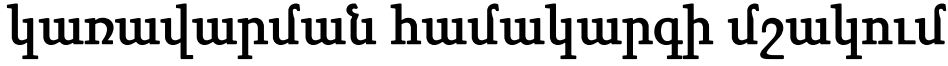

\author{
Uppnıp U. Thunpnujuid l qnıpqti U. Thinnnujud

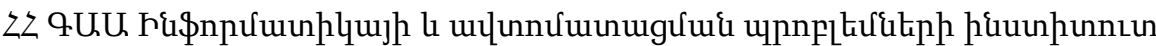 \\ e-mail: arthur@sci.am, gurgen@sci.am
}

\section{Uরựnนุnıu}

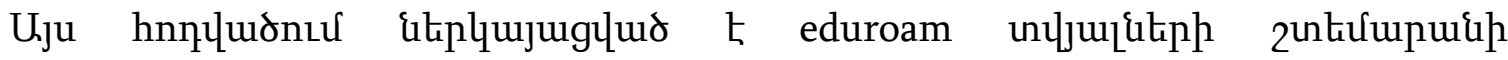

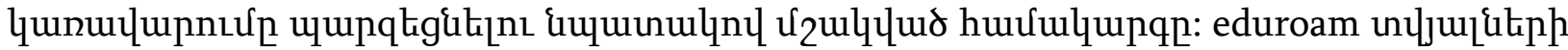

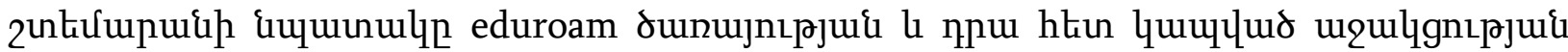

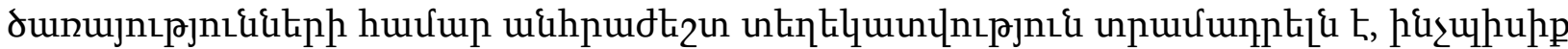

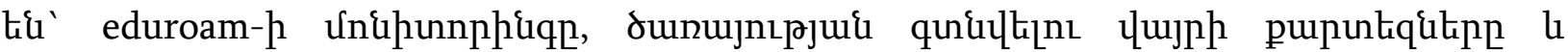

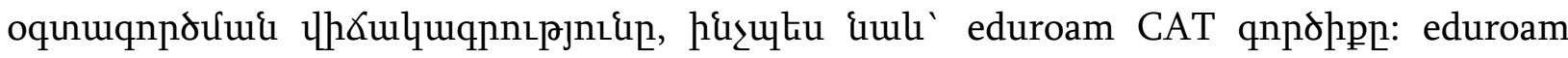

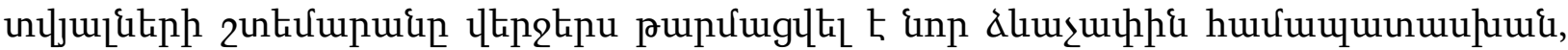

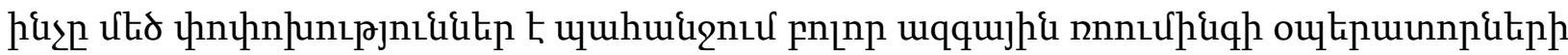

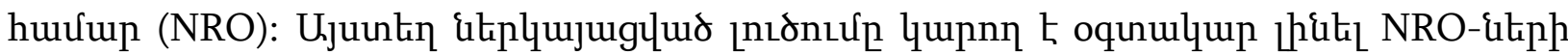
unuhipuunpuunnpitiph hưưu:

Fuquul puntip eduroam, NRO, Wi-Fi, uquup, unihunnphiq,

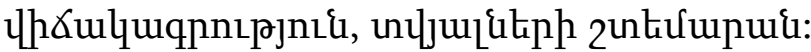

\section{Разработка системы управления обновленной базой данных eduroam}

\author{
Артур С. Петросян и Гурген С. Петросян \\ Институт проблем информатики и автоматизации НАН РА \\ e-mail: arthur@sci.am, gurgen@sci.am
}

\begin{abstract}
Аннотация
В статье представлена система упрощающая управление базой данных eduroam. Цель базы данных eduroam - предоставить необходимую информацию для работы службы eduroam и связанных с ней вспомогательных услуг, таких как мониторинг eduroam, карты расположения служб и статистика использования, а также инструмент CAT eduroam. Спецификации базы данных eduroam были обновлены до второй версии, что требует значительных изменений баз сервиса для всех национальных операторов роуминга (NRO). Представленное здесь решение может быть полезным для администраторов управления базами данных eduroam NRO.
\end{abstract}

Ключевые слова: eduroam, NRO, Wi-Fi, беспроводная связь, мониторинг, статистика, база данных. 